die Äquivalentgewichte der in Frage kommenden Kationen und Anionen, endlich die Quotienten und deren Summen angegeben

\begin{tabular}{|c|c|c|c|c|c|c|}
\hline \multirow{5}{*}{$\begin{array}{l}\text { Calcium-Ion. } \\
\text { Magnesium-Ion } \\
\text { Natrium-Ion } \\
\text { Kalium-Ion . . }\end{array}$} & \multicolumn{3}{|c|}{ In 1000$) \mathrm{ccm}$ Wasser } & Äquiv.-liew: & \multicolumn{2}{|c|}{ Quotienter. } \\
\hline & & . 119,8 & $\mathrm{mg}$ & $1 / 2 \mathrm{Ca}^{*}=20,0$ & 5,99, & \\
\hline & & 32,6 & & $1 / 2 \mathrm{Mg}^{\prime \prime}=12,2$ & 2,67 & \\
\hline & & 42,5 & 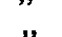 & $\mathrm{Na}=23,0$ & 1,85 & 12,19 \\
\hline & & 65,6 & $"$ & $K^{\prime}=39,1$ & 1,68 & - \\
\hline Hydrocarbonat-I & on. & 468,5 & $\mathrm{mg}$ & $\mathrm{HCO}_{3}^{\prime}=61,0$ & 7,68 & \\
\hline Siulfat-Ion. . . & & 93,0 & & $\mathrm{SO}_{4}^{\prime \prime}=48,0$ & 1,94 & \\
\hline Chlorid-Ion & & 40,2 & ", & $\mathrm{Cl}^{\prime}=35,5$ & 1,13 & 12,23 \\
\hline Nitrat-Ion. & & 94,8 & , & $\mathrm{NO}_{3}{ }^{\prime}=62,0$ & $1,53)$ & \\
\hline
\end{tabular}

Ferro-, Mangano-, Ammonium- und Nitrit-Ion kommen nur dann in Betracht, wenn sie reichlicher zugegen sind. Kinselsäure ist zwar in jedem Wasser vorhanden, doch ist dies bei der Prüfung der Analysenergebnisse auf ihre Richtigkeit belanglos, da bei der Bestimmung der Alkalinität die Kieselsäure auf Methylorange wirkungslos ist; ebenso bleibt die im Wasser enthaltene freie Kohlensüur" unberücksichtigt.

$$
\text { Zusammenfassung. }
$$

Vin bei der Aufbewahrung stark alk a lischer Lös u u ge n das Einkitten des Glasstöpsels zu verhindern, wird (inil G l ycerinzusatz empfohlen.

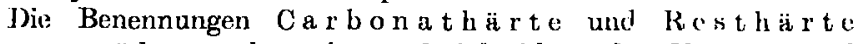
bzw. vor übergebende und bleibende $\mathrm{H} \ddot{a} \mathbf{r t e}$ sind streng auseinander $\mathrm{zu}$ halten.

Es wurden für Wasseruntersuchungen dienende Verfahren angegeben, die den $\mathrm{Nach}$ we is und die Bestimmung geringer Mengen von $\mathrm{Brom}$, Jod und Arsen gestatten.

Hat man eine e rs c höpfende $W$ asseranalyse ausgeführt, so ist es im allgemeinen nützlich, das Ergebnis auf seine Richtigbeit rechnerisch \% u prïfen.

\section{Die Änderungen des deutschen Patentgesetzes nach den Erfahrungen des Weltkrieges.}

\section{Von Patentanwalt Dr. Juliugs Erraarm, Berlin.}

(Fortsetzung statt Schinß von S. 1i2.)

Auch die Patento auf Herstellung pharmazeutisch verwendbarer Stoffe können durch eine zu enge Auslegung des Schutzes ruf die Frzeugnisse in Mitleidenschaft gezogen werden. Nach den Ankündigungen in Frankreich kann man erwarten, da $B$ die Patente auf die Herstellung pharmazeutischer Produkte noch mehr eingeengt werder, wie dies schon unter dem geltenden Patentgesetze der Fall ist, und daß ein Konkurrenzkampf mit Deutschland auf pharmazeutischon Gebiete beabsichtigt ist. Durch die Wahl besonderer Arzneiformen kann dieser Kampf besonders geführt werden. Hierbei würdle es sich um eine Vermischung des Verfahrenserzeugnisses mit anderen Stoffen handeln, so daß also im Sinne der Strafsenate des Reichsgerichts keine unmittelbaren Errzeugnisse vorliegen würden. Dic Arzneiformen könnten also nach I (utschland eingeführt werdon.

Der Schutz der Erzeugnisse hat noch nach anderer Richtung Bedeutung. Bereits jetzt hat die Industrie der künstlichen plastischen Massen wie Acetylcellulose, Galalith u. dgl. besondere Wichtigkeit. Im Kriege hat man sich im großen Umfange an die Benutzung von Crsatzstoffen für Naturprodukte gewöhnt, zu welchem Zwecke die plastischen Massen besonders Verwendung finden. Die Herstellung von Gegenständen aus dø $n$ Massen erfolgt vielfach im Auslande, von wo aus die verarbeitete'، Erzeugnisse, z. B. Känume, die einen großen Handelsartıl'el bilden;, nach Deutschland eingeführt werden. Hierdurch kann d is $\mathbf{R}_{\mathrm{c}}$ ht des deutschen Patentinhabers beeinträchtigt verden, falls das Material der Gegenstände, also die plastischen Massen, im Auslande unter Verletzung der Patentrechte hergestellt werden. Mit derartigen Möglichkeiten muß aber in Zukunft eben gerechnet werden. Dementsprechend ergibt sich die Notwendigkeit, der Bestimmung eine Fassung zu geben, welche die Fälle deckt, in denen die Bearbeitung zwar die brauchbare Form gibt, das Material aber doch dem Gegenstande den Stempel aufdrückt und die Verwendbarkeit, wenn nicht ausschließlich, doch wenigstens zum großen Teil, vielleicht sogar ausschlaggebend beeinflußt. Die Fassung wird Sehwierigkeiten bereiten, doch können Bedenken, wie sie für die Reichstagskommission 1891 maßgebend waren, eine zu weite Ausdehnung des Schutzes zu verhindern, heute kaum noch in Betracht kommen. Die Furcht, daß der Patontschutz zu großen Umfang erlangen würde, und die Freiheit des Verkehrs in Gefahr käme, dürfte sich allmählich verloren haben. Außerdem kommt in Betracht, daß auch der deutsche Patentinhaber zur deutschen Industrie gehört, und der ihm zuteil werdende Schutz wiederum der gesamten deutschen Industrie zugute kommt. Die deutsche Industrie würde also auch durch die Stärkung der Stellung des deutschen Patentinhabers gefördert werden, selbst wenn ein anderer deutscher Mitbewerber Unbequemlichkeiten durch das Bestehen des Patentschutzes erleiden würde. Unter diesen Umständen kann man ohne Bedenken auf dic Regierungsvorlage von 1891 zurückgreifen und den Schutz aus dem Verfahrenspatente allgemein auf die Erzeugnisse nach demselben erstrecken, indem man die Beschränkung auf die ,unmittelbaren“" Erzeugnisse fallen läßt. Man käme dann beispielsweise zu der Fassung:

"Ist das Patent für ein Verfahren erteilt, so erstreckt sich die Wir. kung auch auf die durch das Verfahren, gleichgültig ob dasselbe in einem Herstellen, Bearbeiten oder Verarbeiten besteht, erhaltenen Erzeugnisse".

\section{Der Schutz von Verfahren zur Herstellung neuer $S t$ of $f$.}

Um die Beweislast für die behauptete Verletzung von Patenten auf die Herstellung neuer Stoffe zu erleichtern, ist bestimmt, da B bis zum Beweise des Gegenteils jeder Stoff gleicher Beschaffenheit als nach dem patentierten Verfahren hergestellt gilt (Patentgesetz 1891, § 35 Absatz 2, Vorentwurf $\$ 47$ Absatz 3). Diese Bestimmung über die Beweisvermutung ist im Gesetze bei der Behandlung der Schadensersatzpflicht angeführt und bezieht sich also nur auf dic: Zivilverfolgung von Patentverletzungen. Da die Strafandrohung keine derartige Bestimmung über die Beweisführung enthält, gilt in Strafverfahren nicht die angeführte Beweiserleichterung. Das Strafverfahren wegen Patentverletzungen ist hiernach namentlich bei chemischen Erfindungen, für welche die Beweiserleichterung in erster Linie bestimmt ist und besondere Bedeutung hat, wesentlich schwieriger als bei der Zivilklage. Soweit es sich um Verletzungen handelt, bei denen ausschließlich Deutsche beteiligt sind, führt dies im allgemeinen zu keinen wesentlichen Úbelständen, denn im allgerneinen kommt man auch durch die Zivilklage zum Ziel. Anders ist aber die Sachlage, wenn das patentierte Verfahren im Auslando ausgeführt wird, und die patent verletzende Handlung dadurch begangen wird, da $B$ die Erzeugnisse in Deutschland vertrieben werden. Die Mittelsperson, welche die Einführung in Deutschland und del Verkauf besorgt, ist meist mittellos, so daß man mit einer Schadens ersatzklage nichts erreicht. Auch wenn die Unterlassungsklage zun Siege führt, nutzt dies im allgemeinen wenig, denn es übernimmt dann eine andere Person den Vertrieb. In derartigen Fällen bleibt tatsächlich nur die Strafverfolgung übrig, wie auch die seltenen Fälle der Verurteilung zu Gefängnis wegen Patentverletzung ein derartiges Vorkommnis betrafen. Die Straf verfolgung ist aber dadurch erschwert und meist sogar unmöglich gemacht, daß der Beweis über die tatsächliche Anwendung des patentierten Verfahrens nicht geführt werden kann, weil ja die Ausführung im Auslande geschieht. Das einzige Hilfsmittel, um die Durchführung des Strafverfahrens und die Ahndung der Patentverletzung zu erreichen, besteht in der Ausdehnung der Beweisvermutung von der Zivilklage auf die Straf verfolgung. Die Gesetzesfassung kann keine Schwierigkeiten bieten, sobald man sich über die grundsätzliche Frage geeinigt hat.

Das einzige Bedenken, das gegen die Ubernahme der Beweisvermutung in die Strafbestimmungen geltend gemacht werden kann, besteht darin, daß eine ähnliche Beweisvermutung dem Strafgesetzc und dem Strafverfahren fremd ist. Diese Erwägungen können aber nicht gegen die Anwendung einer Bestimmung sprechen, wenn nux dieses eine Mittel zur Rechtsdurchsetzung geeignet ist. Die Patentverletzung nimmt unter den Delikten überhaupteine Sonderstellung ein, so daß auch mit Rücksicht hierauf eine besondere Bestimmung zur Ermöglichung der Bestrafung nicht abgelehnt werden kann. Der Angeklagte ist auch durch die Annahme der Beweisvermutung durchaus nicht in seiner Verteidigung übermäßig beschränkt. Er kann sich der Beweisvermutung einfach dadurch entziehen, daß er das zur Herstellung des neuen Stoffes tatsächlich benutzte Verfahren bekannt gibt und den Nachweis über den Nichteingriff desselben in das patentierte Verfahren führt. Man kann auch nicht don Einwand erheben, daß der Importeur und der Agent oder der Kommissionär das Herstellungsverfahren nicht kennen. Gerade dieser Einwand soll durch die Beweisvermutung getroffen werden. (SchluB folgt.) 